\title{
A Genome Scan for Eye Color in 502 Twin Families: Most Variation is due to a QTL on Chromosome 15q
}

\author{
Gu Zhu', David M. Evans', David L. Duffy', Grant W. Montgomery', Sarah E. Medland', Nathan A. Gillespie', \\ Kelly R. Ewen'2, Mary Jewell ${ }^{3}$, Yew Wah Liew 4 , Nicholas K. Hayward', Richard A. Sturm ${ }^{5}$, Jeffrey M. Trent', \\ and Nicholas G. Martin' \\ ' Queensland Institute of Medical Research, Brisbane \\ ${ }^{2}$ Australian Genome Research Facility, Melbourne \\ ${ }^{3}$ Center for Inherited Disease Research, Baltimore \\ ${ }^{4}$ Australian Red Cross Blood Service, Brisbane \\ ${ }^{5}$ Institute for Molecular Bioscience, University of Queensland, Brisbane \\ ${ }^{6}$ National Cancer Institute, Bethesda, and Translational Genomics Research Institute, Phoenix
}

W e have rated eye color on a 3 -point scale (1 = blue/grey, 2 = hazel/green, 3 = brown) in 502 twin families and carried out a 5-10 cM genome scan (400-757 markers). We analyzed eye color as a threshold trait and performed multipoint sib pair linkage analysis using variance components analysis in Mx. A lod of 19.2 was found at the marker D15S1002, less than $1 \mathrm{cM}$ from OCA2, which has been previously implicated in eye color variation. We estimate that $74 \%$ of variance in eye color liability is due to this QTL and a further $18 \%$ due to polygenic effects. However, a large shoulder on this peak suggests that other loci affecting eye color may be telomeric of OCA2 and inflating the OTL estimate. No other peaks reached genome-wide significance, although lods $>2$ were seen on $5 p$ and $14 q$ and lods $>1$ were additionally seen on chromosomes 2, 3, 6, $7,8,9,17$ and 18. Most of these secondary peaks were reduced or eliminated when we repeated the scan as a two locus analysis with the $15 \mathrm{q}$ linkage included, although this does not necessarily exclude them as false positives. We also estimated the interaction between the $15 \mathrm{q}$ OTL and the other marker locus but there was only minor evidence for additive $x$ additive epistasis. Elaborating the analysis to the full two-locus model including non-additive main effects and interactions did not strengthen the evidence for epistasis. We conclude that most variation in eye color in Europeans is due to polymorphism in OCA2 but that there may be modifiers at several other loci.

Skin, hair and eye colors are some of the most obvious forms of human diversity (Sunderland, 1956), yet the complexities of the genetic mechanisms underlying the differences of these pigmentation phenotypes within individuals remain largely unknown. It is expected that a relatively small set of major genes contribute to the color variation of these cutaneous tissues within diverse human populations. These pigmentary genes are also expected to be specifically expressed within the melanocyte cell and act to regulate the synthesis, chemical composition, packaging and distribution of the pigment melanin, thus producing the visible differences underlying these traits (Sturm et al., 1998; Sturm et al., 2001).

The iris consists of several layers of which the most important for the appearance of eye color are the anterior layer and its underlying stroma (Robins, 1991; Boissy, 1998). In the brown iris there is an abundance of melanocytes and melanosomes in the anterior layer and stroma. In individuals with blue eye color, the anterior layer and stroma contain very little melanin. As light traverses these relatively melanin-free layers, the minute protein particles of the iris scatter the short blue wavelengths to the surface. The blue color is heightened because longer wavelengths (yellow and red) are absorbed by the dark background of the pigmented retinal epithelium which also obscures the reddish hue of the adjacent blood vessels. Other colors such as yellow, green and hazel are due to varying amounts of lipid pigment granules in the iris. With respect to eye color inheritance, blue is usually accepted as being recessive to brown eyes but this is not always the case and it seems likely that more than one gene is involved (McKusick, Online Mendelian Inheritance in Man database \#227240). However, in a study of a representative United States Caucasian population, the $55 \%$ of individuals with blue eyes were

Received 17 December, 2003; accepted 4 February, 2004.

Address for correspondence: Nick Martin, Queensland Institute of Medical Research, Brisbane Qld 4029, Australia.Email: nickM@qimr.edu.au 
assumed to be $b b$ homozygotes, from which the frequency of the dominant brown eye allele $B$ was estimated to be $26 \%$ (Hasstedt, 1995). Recently, Larsson et al. (2003) assessed ratings of eye color in 199 pairs of adult twins and confirmed high correlations of .87 and .86 in male and female monozygotic (MZ) pairs respectively, with corresponding dizygotic (DZ) correlations of .26 and .43. However, they estimated the DZ opposite sex correlation at .69, which seems anomalously high, and therefore drove them to conclude that there was shared environmental influence on eye color rating, which seems counterintuitive.

The leading candidate gene for human eye color is the OCA2 gene which is mutated in Type II oculocutaneous albinism and encodes the P-protein (Brilliant, 2001). It has recently been suggested that it is a major human pigmentation gene determining skin color through interaction with the melanocortin-1 receptor (MC1R) signalling pathway (Akey et al., 2001; Ancans et al., 2003; Duffy et al., 2004). Moreover, polymorphism of the OCA2 gene almost certainly underlies the previous assignment of the brown eye (BEY2/EYCL3, MIM227220) and brown hair (HCL3, MIM601800) loci on chromosome 15q (Eiberg \& Mohr, 1996). Two OCA2 coding region variant alleles, Arg305Trp and Arg419Gln, were recently shown to be associated with brown or green/hazel eye colors respectively (Rebbeck et al., 2002). To date, however, there have been no systematic microsatellite-based linkage searches for genes affecting eye color variation. To address this issue we estimated heritability and conducted a genome-wide linkage scan in a sample of 502 twin families assessed for eye color.

\section{Method \\ Participants}

Twins were recruited in the context of an ongoing study of melanoma risk factors, including hair, skin and eye color and the development of melanocytic naevi (moles). The clinical protocol has been described in detail elsewhere (Aitken et al., 1996; McGregor et al., 1999; Zhu et al., 1999). Twins were enlisted by contacting the principals of primary schools in the greater Brisbane area, media appeals and by word of mouth. It is estimated that approximately $50 \%$ of the eligible birth cohort were recruited into the study. The sample appeared representative with respect to mole count (Zhu et al., 1999) and IQ (Wright et al., 2001) and it seemed reasonable, therefore, to suppose that the sample was also representative of the Queensland population with respect to eye color. Informed consent was obtained from all participants and parents prior to testing. This paper concerns data collected from May 1992 to February 2001.

Eye color and genotyping were available for 502 twin families (419 DZ, 83 MZ). Genotypes were available for both parents in 358 of these families, for one parent only in 96 families, and for neither parent in 48 families. Eye color was also collected for some parents but is not used in the analysis here. One or more extra siblings were both phenotyped and genotyped in 149 DZ families and, of necessity for linkage analysis, all $83 \mathrm{MZ}$ families. The number of offspring with unique genotypes (i.e., omitting $\mathrm{MZ}$ co-twins) was 1205 (1025 from DZ families, 180 from MZ families).

\section{Assessment of Eye Color}

Eye color was rated on a 3 -point scale ( $1=$ blue/grey, 2 = hazel/green, 3 = brown) by the same research nurse (AE) for all twin and sibling participants. Most twins who were tested at age 12 were also tested at 14 and some again at 16 , so for many participants there were two and even three ratings of eye color. For 826 twins rated at both 12 and 14 years of age, all but $39(4.7 \%)$ had consistent ratings on two occasions; all inconsistencies were between adjacent points on the scale (i.e., between 1 and 2, or 2 and 3). For the purposes of linkage analysis, these were handled in two ways: (a) subjects were given the rating assigned on their first visit; and (b) these subjects were all rated as 2, on the grounds that inconsistency indicated that they did not fall into one extreme category (blue) or the other (brown). Linkage scans under the two treatments of rating inconsistency were compared.

There are several ways in which our eye color data can be handled statistically. One is to treat the categories as nominal and analyse three dichotomous variables, namely, blue/grey versus other, green/hazel versus other, and brown versus other. This is the approach used by Eiberg and Mohr $(1987,1996)$ in their first analyses of eye color linkage. In the present paper we have chosen to view eye color as a continuum, on the grounds that it is largely a function of quantitative differences in the density (Eagle, 1988; Imesch et al., 1996) and size (Eagle, 1988) of melanosomes, and possibly also the quantity or quality of melanin within the melanosomes. It is also possible that the amount of eumelanin versus pheomelanin or the ratio of brown-soluble to black-insoluble forms of eumelanin varies between different colored irises. We therefore view the three color categories as ordered on a continuum which, for statistical convenience, we assume to be a normally distributed trait. This is not an unreasonable assumption if eye color is determined by many different genes, in which case the central limit theorem leads us to expect a distribution tending toward normal. As we shall see, however, most variation appears to be due to a single major locus (though possibly with many different functional alleles), and this may result in serious departures from bivariate normality.

\section{Zygosity Testing and Genotyping}

Genomic DNA was extracted (Miller et al., 1988) from peripheral venous blood samples. Before selection for genome scanning, zygosity of same-sex twins was determined by typing nine independent DNA microsatellite polymorphisms plus the sex marker 
amelogenin at the Queensland Institute of Medical Research (QMIR), Brisbane, using the Profiler multiplex marker set (AmpFLSTRR Profiler PlusT, Applied Biosystems, Foster City, CA). All twins were also typed for $\mathrm{ABO}, \mathrm{Rh}$ and MNS blood groups by the Red Cross Blood Service, Brisbane. Genome scans were carried out at the Australian Genome Research Facility (AGRF), Melbourne, and the Center for Inherited Disease Research (CIDR), Baltimore. At AGRF, PCR was performed on $30 \mathrm{ng}$ of DNA and genotyping completed using ABI PRISM* 377 sequencing machines (Ewen et al., 2000). Dye-labelled PCR products were detected using GENESCAN (CPE Applied Biosystems) software and allele calling was performed using Genotyper 2.1 (PE Applied Biosystems). The same methods were used at CIDR except $40 \mathrm{ng}$ of DNA was used and allele calling used Genotyper 2.5 (Weeks et al., 2002).

\section{Details of Genome Scans}

Three genome scans were performed on blood samples collected from twins, siblings and parents through the course of the adolescent twin study. Numbers in this section are for the entire study sample of 525 families, although only 502 families have sufficient eye color data to be included in the statistical analysis in this paper. Use of the full sample improves allele frequency estimates for IBD estimation. The relationships between the subsets of individuals in the three scans are shown in Figure 1 and the relationships between the three marker sets in Figure 2. The first scan was performed by AGRF and consisted of 382 autosomal and $18 \mathrm{X}$-chromosomal markers at an average spacing of $9.1 \mathrm{cM}$ (Figure 3). The scan was performed on the twins and siblings from the first 274 families (642 individuals) enrolled in the study (note that no parents were typed during this phase).

The second genome scan was performed by CIDR and consisted of 370 autosomal, 16 X-linked markers and $3 \mathrm{Y}$ markers at an average spacing of $9.4 \mathrm{cM}$ (Figure 3). The scan was performed on 493 families (1,999 individuals) which comprised 242 families (518 individuals) from the first AGRF scan, 180 "new" families (1,237 individuals) and involved parents as well as twins and siblings. Thirty-five markers from the CIDR and AGRF scans were shared. The third genome scan was performed by the AGRF on 71 families (244 individuals) from the CIDR dataset who were not genotyped in the first AGRF scan, and from whom genotypes were unavailable from both parents. The rationale behind this decision was that increased marker density should result in a large gain of information since parental genotypes were unknown for these individuals. In contrast, it was thought that little would be gained from additional genotyping of individuals for whom parental genotype data were already available, since IBD status would already be known with a high degree of certainty. The markers used in this scan were identical to the markers used in the first AGRF scan, save that additional new markers were

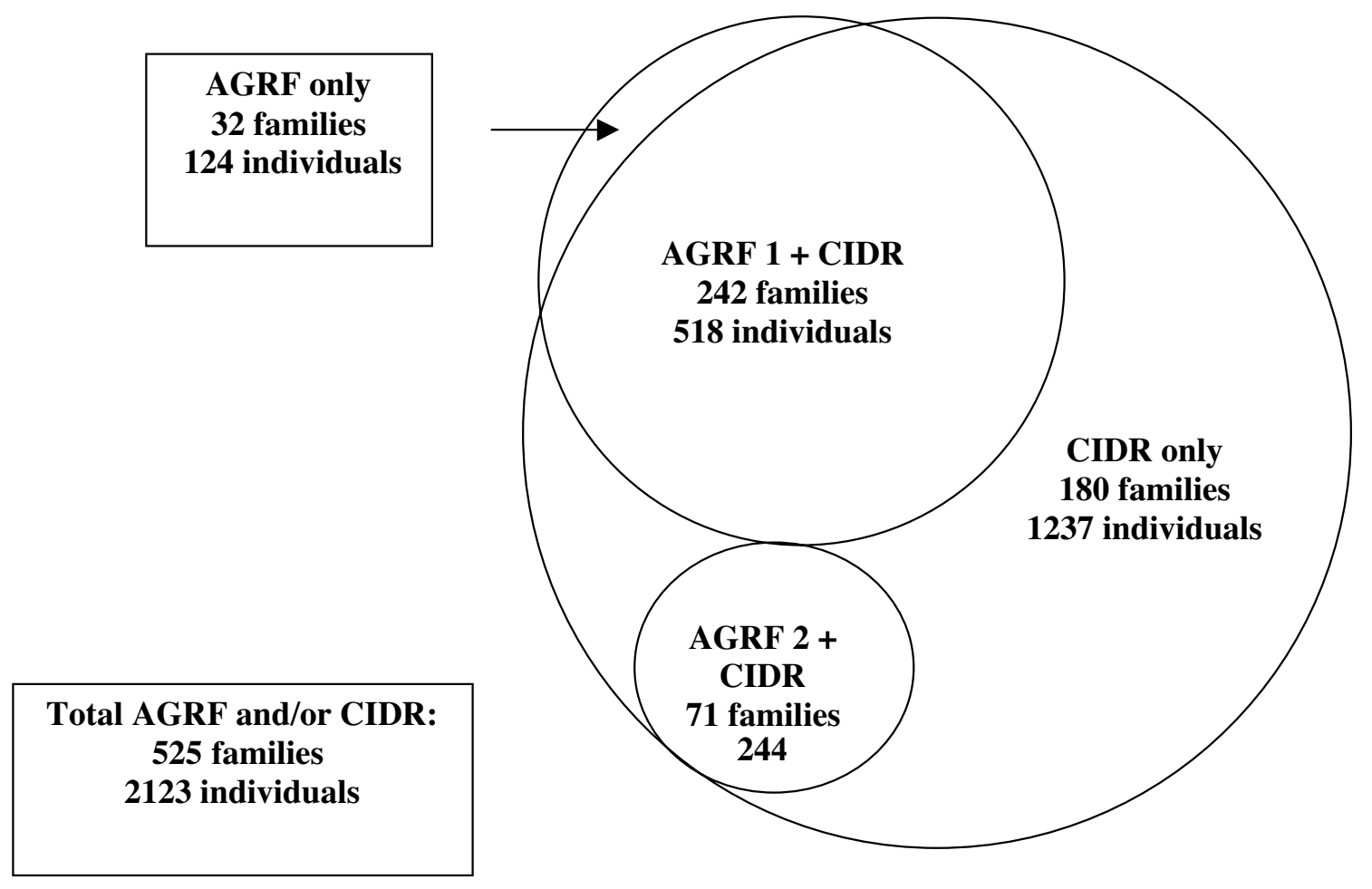

Figure 1

Venn diagram illustrating the breakdown of participants from the three genome scans. 


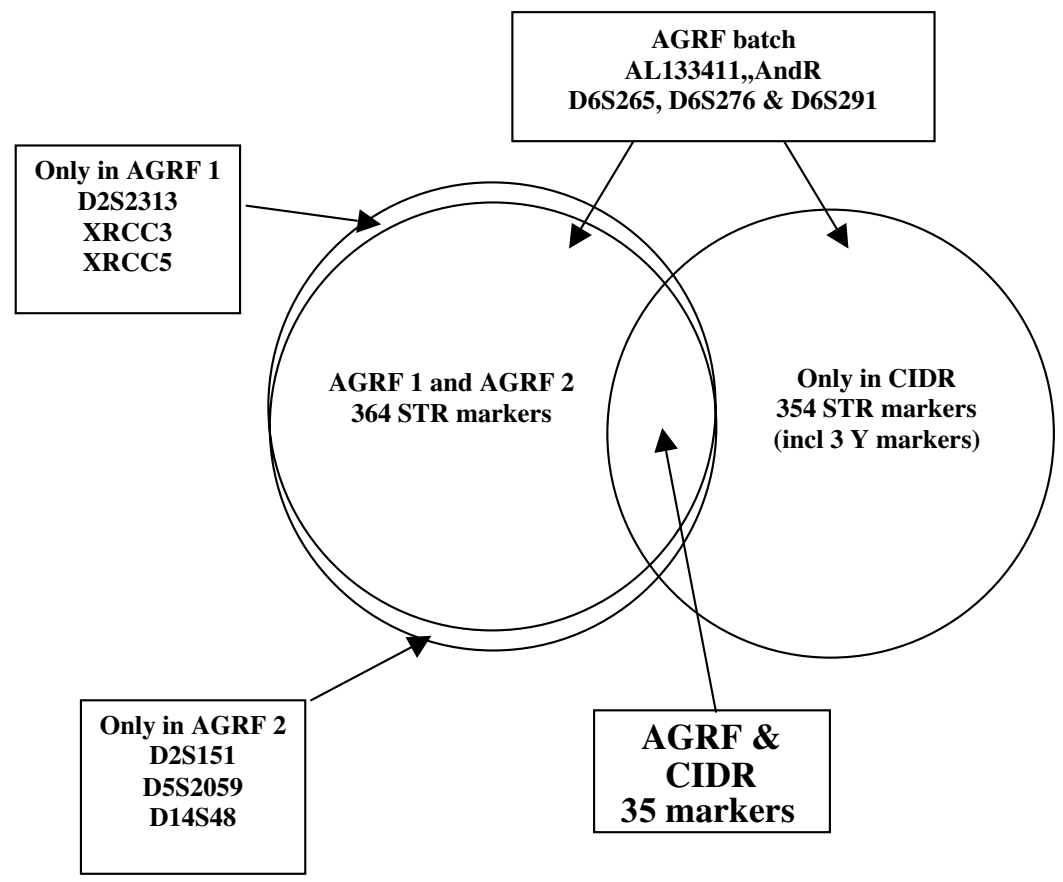

Figure 2

Venn diagram showing the relationship between the marker sets used in the three genome scans.

typed on chromosomes 2 (D2S151), 5 (D5S2095) and 14 (D14S48), and a marker on chromosome 2 was not typed (D2S2313; see Figure 2). Finally, five new markers (three on chromosome 6 adjacent to the MHC loci, one on chromosome 9p, and the XAR [CAG]n repeat on the $\mathrm{X}$ chromosome) were typed on all samples at AGRF and integrated in the marker map for the genome scan. Marker locations were determined from the deCODE map (Kong et al., 2002; Leal, 2003).

\section{Cleaning Genotype Data}

The first stage of data cleaning involved making sure that the relationships specified in the pedigree files were accurate using RELPAIR (Boehnke \& Cox, 1997; Epstein et al., 2000) and Graphical Representation of Relationships (Abecasis et al., 2001a). These programs detect relationship misspecifications (e.g., non-paternities, MZ twins, sample mix-ups), but through slightly different methods. RELPAIR calculates the multipoint likelihood of the marker data for each pair conditional on each of four possible relationships (i.e., MZ twins, full siblings, half siblings and unrelated pairs). The likelihood is a function of the usual parameters (i.e., marker allele frequencies, inter-marker distances) and also the presumed relationship of the pair. The inferred relationship is the one that maximizes the likelihood (Boehnke \& Cox, 1997; Epstein et al., 2000).

In contrast, Graphical Representation of Relationships (GRR) is a Windows-based application designed to detect pedigree errors by displaying the distribution of marker allele sharing (from identity by state rather than identity by descent) among pairs of individuals, both within and across pedigrees. The different kinds of relationships (e.g., full sibs, half sibs, MZ twins, parent-offspring) each have a characteristic expected mean and variance of IBS sharing. For example, MZ twins are expected to share two alleles IBS with a variance of zero (since they will share two alleles across all loci), and so can be quite easily distinguished from full siblings who are roughly expected to share one allele IBS with variance one half, and from parents who are expected to share one allele IBS with variance zero (exact values will depend on how polymorphic the marker is). GRR may also have an advantage over the program RELPAIR in that it will identify MZ twin pairs who share most but not all of their alleles IBS (presumably because of genotyping error or somatic mutation). In contrast, these pairs may not be flagged by RELPAIR since they do not share all their marker alleles IBS. (Note that Epstein et al., 2000, claim that this oversight has been rectified in 
the latest version of RELPAIR.) GRR is also useful in detecting sample mix-ups across pedigrees by examining the distribution of allele sharing amongst all pairs of individuals (i.e., not just within families).

The second stage of data cleaning involved deleting genotypes which gave rise to Mendelian inconsistencies. This was achieved using two programs with Mendelian error checking routines. The program Sib-pair (Duffy, 2002) was used to correct autosomal data, but as this program cannot handle data from X-linked loci, the program ASPEX (Davis $\&$ Weeks, 1997) was used for this chromosome.

The next step in data preparation was to merge marker data from the three genome scans. As mentioned, 35 markers were shared between CIDR and AGRF genome scans. Since the genotypes at these shared loci should be identical from both scans, this was another opportunity to identify sample mix-ups and typing errors. The small number of genotypes

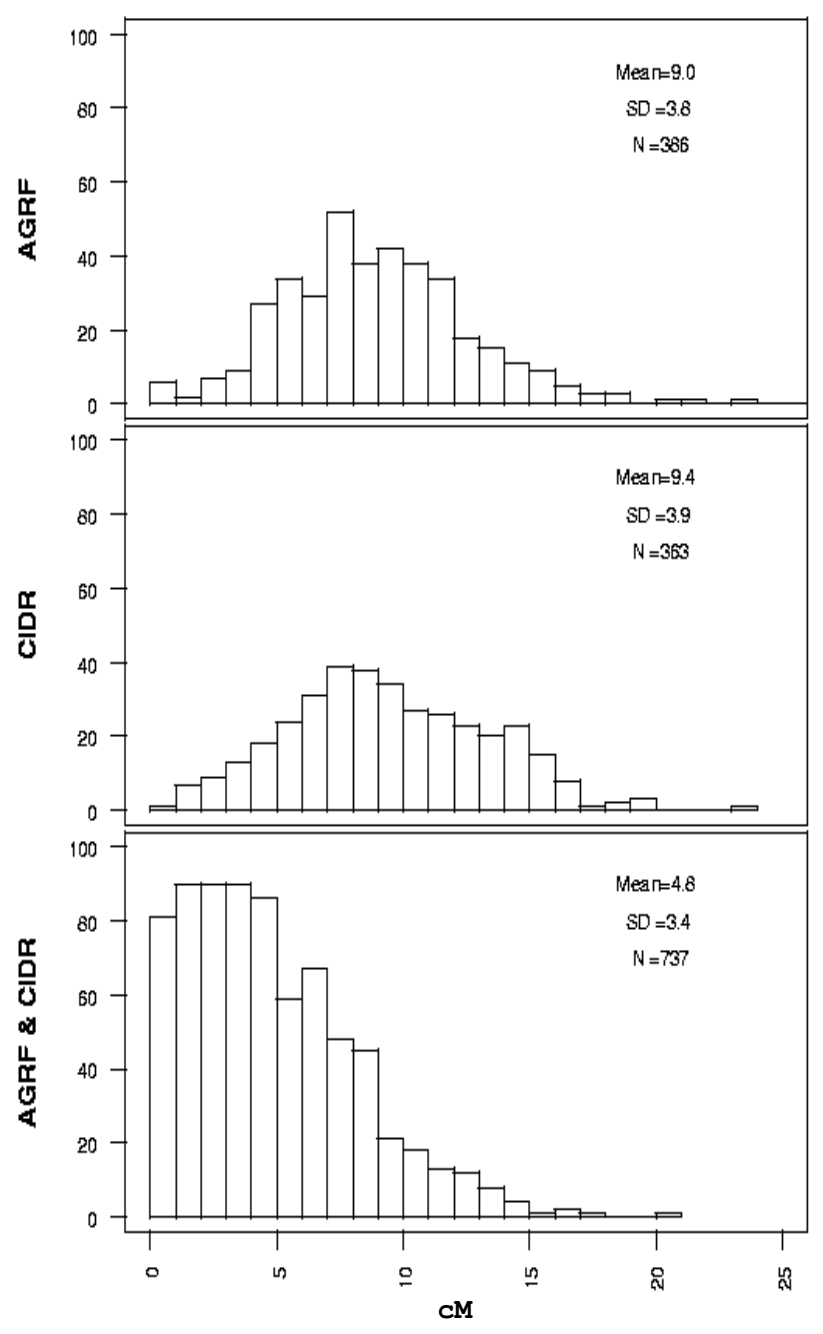

Figure 3

Distribution of intermarker distances in (a) AGRF, (b) CIDR, and (c) combined AGRF + CIDR genome scans according to the DECODE genetic map (Kong et al., 2002). which were inconsistent between scans were deleted from the dataset. However, three loci (markers D3S1304, D4S403 and D4S391) contained an unusually high number of inconsistencies between CIDR and AGRF scans. Since it was impossible to know which scan contained the "correct" genotypes, it was decided not to merge data from these markers. Instead, we included data from both scans in the analysis, with the markers separated by an arbitrarily small distance $(0.2 \mathrm{cM})$, the rationale being that any serious errors would be detected as "double recombinants" at the next stage of cleaning.

Next, the combined dataset was examined for "Mendelian consistent" errors which typically manifest themselves in the form of spurious "double recombinants". Since this is very unlikely to occur at closely spaced loci (a fortiori because of interference), it is much more likely the result of genotyping error. Many laboratories are content to detect and delete Mendelian inconsistent errors only. However, it is well known that both types of genotyping error can severely affect the power to detect linkage (Abecasis et al., 2001b; Douglas et al., 2000). Since Mendelian consistent errors are thought to constitute greater than $25 \%$ of mistypings in nuclear family data (Douglas et al., 2000; Sobel et al., 2002), their impact should not be under-estimated. One strategy is to use a haplotyping program such as ALLEGRO (Gudbjartsson et al., 2000) to identify double recombinants and then to delete the problematic cases from the dataset. The weakness of this approach is that these programs only provide a single (although the most likely) haplotype configuration. They provide no indication of how likely the haplotype is and whether there are many equally likely or slightly less likely configurations consistent with the data (Sobel et al., 2002).

The approach we took was to employ Analysis option III of the MENDEL software package (Lange et al., 2001) which computes the posterior probability that an individual's genotype is incorrectly specified as well as the probability of there being a genotype error (anywhere) in the pedigree at that locus. This probability depends upon the parameters in the likelihood function (i.e., the allele frequencies and marker map distances) and also a penetrance function which specifies how the observed genotypes are related to hidden "true" genotypes (Sobel et al., 2002). In this way the likelihood of the haplotype is incorporated in the calculation of the total posterior probability.

In order to minimize the impact of genotyping error (but without being overly conservative) a posterior probability of $80 \%$ was arbitrarily chosen as the criterion for which a genotype was considered an error. That is, an individual's genotype was deleted at a locus if the posterior probability of there being an error was greater than or equal to $80 \%$. In cases where there was an $80 \%$ or greater probability of an error in the pedigree, but the particular individual responsible could not be determined with certainty 
(i.e., with $>80 \%$ probability), marker information for the entire pedigree was deleted at that locus.

The final procedure for error checking consisted of performing a series of 2-point parametric linkage analyses at flanking markers to loci coding for three Mendelian phenotypes whose map positions are known (ABO, Rhesus, and MNSs blood groups). Analyses were performed using the ILINK and MLINK subroutines from the LINKAGE package of programs (Lathrop et al., 1984). ILINK was run to find the maximum lod score (MLOD) and recombinant fraction at each marker, and then MLINK was run at a recombination value of zero in order to identify any individuals who were recombinant between marker and blood group loci. Analysis of linkage between the MNSs blood group system and the flanking markers D4S1625 (CIDR) and D4S1629 (CIDR) revealed highly significant linkage $(\mathrm{MLOD}=105.79$, $\theta=.015$, and MLOD $=25.67, \theta=.136$ respectively) . The MLINK option revealed 11 individuals who were recombinant between D4S1625 and MNSs, while 74 individuals were recombinant between MNSs and D4S1629 (which is located approximately $11 \mathrm{cM}$ downstream from MNSs). Highly significant linkage was found between D1S234 (AGRF; MLOD $=41.03$, $\theta=.032), \mathrm{D} 1 \mathrm{~S} 1622(\mathrm{CIDR} ; \mathrm{MLOD}=69.97, \theta=.035)$ and the eight haplotypes arising from the $\mathrm{D} / \mathrm{d}$ and $\mathrm{CE} / \mathrm{Ce} / \mathrm{cE} / \mathrm{ce}$ polymorphisms of the Rhesus blood group. MLINK analyses revealed 8 individuals who were recombinant between Rh and D1S234 and 20 individuals who were recombinant for D1S1622. These results reflect the relative informativeness of the family structures at each locus; while there was greater evidence for linkage between $\mathrm{Rh}$ and D1S622, this marker was located approximately $3 \mathrm{cM}$ downstream from $\mathrm{Rh}$, while D1S234 was located approximately $0.5 \mathrm{cM}$ upstream. Similarly, a high degree of linkage was found between D9S164 (AGRF; MLOD = 39.19, $\theta=.001), \mathrm{D} 9 \mathrm{~S} 2157$ (CIDR; MLOD $=63.01, \theta=.009$ ) and the $A_{1} / A_{2} / B$ polymorphisms of the $A B O$ blood group. Two individuals were recombinant between $\mathrm{ABO}$ and D9S2157, no recombinants were found at the D9S164 locus. The presence of recombination between two very tightly linked loci may indicate that a sample mix-up has occurred. Thus, the fact that there were no more recombinants than would be expected (given the distance between loci, the informativeness of the family structures at the markers and the information content of the markers) and that these individuals were not flagged more than once, suggests that no sample mix-ups were present.

The result of merging the genome scans after cleaning (AGRF \& CIDR) was a dense map consisting of 796 markers at an average spacing of $4.8 \mathrm{cM}$ in Kosambi units (Figure 3c). Ordering of markers was based on the physical map and genetic map distances were based on the deCode map with interpolation of unmapped markers. The one large intermarker distance is located on the q telomere of the $\mathrm{X}$ chromosome.

\section{Statistical Analysis}

Standard methods for maximum likelihood analysis of raw ordinal data were used (Neale et al., 2002). In our case, the observed values were eye color categories on a 3-point scale. Their expected values were estimated based on the bivariate normal distribution in which the underlying normal scale of liability to light/dark eye color was trichotomised by two thresholds which were estimated simultaneously with the components of variance hypothesized to account for the correlation in liability between twins and sibs (Duggirala et al., 1997). This correlation was parameterized in the usual way as a function of variance due to a linked QTL $(\mathrm{Q})$, to a residual polygenic effect (A), and to unique environmental variance, including measurement error (E). Shared environment (C) may also be included in the model, although it is not expected to affect eye color, unless through rater bias. With DZ twins and sibs only, we were unable to resolve $\mathrm{A}$ and $\mathrm{C}$ and they were estimated as a residual familial effect, F. Since we have some MZ pairs who also have sibs who are phenotyped and genotyped in our linkage sample, formally we can resolve A and C, although we have little power to do so. In practice, all we were interested in was the size and significance of the QTL effect Q, at each marker, so for the present purpose we omitted the data for the second $\mathrm{MZ}$ twin and parameterized our model as FEQ.

Structural equation modelling analyses were performed using the Mx software package (Neale et al., 2002). Identity by descent estimation probabilities at each of the markers were estimated using MERLIN (Abecasis et al., 2002).

\section{Linkage Analyses}

Univariate multipoint variance components (VC) linkage analysis was used to test for linkage between marker loci and eye color (Almasy \& Blangero, 1998; Amos, 1994; Eaves et al., 1996; Fulker \& Cherny, 1996). The expected phenotypic covariance matrix for sibs (or DZ twins) may be written as $\Sigma=\hat{\Pi} \mathrm{Q}+\mathrm{F}+\mathrm{E}$, where $Q$ is the additive genetic variance due to the QTL which is linked to the genotyped marker, F is the residual familial covariance which can only be resolved into polygenic additive genetic variance (A) and shared environment $(\mathrm{C})$ if $\mathrm{MZ}$ twins are included in the analysis, and $\mathrm{E}$ is the unique environmental variance. $\hat{\Pi}$ is a matrix containing the elements $\hat{\pi}_{i j}$ which denote the estimated proportion of alleles shared IBD at the trait locus by individuals $i$ and $j$ (estimated as $\left.\mathrm{P}[\mathrm{IBD}=2]+{ }_{1} / 2 \mathrm{P}[\mathrm{IBD}=1]\right)$. X-linked variance components linkage analysis was performed using the MINX program of Abecasis (http://www.sph.umich.edu/csg/ abecasis/Merlin/reference.html).

The null hypothesis that additive genetic variance caused by a QTL linked to a marker for a given phenotype is zero (i.e., $\mathrm{Q}=0$ ) was tested against a model in which $\mathrm{Q}$ is free. Twice the difference in natural $\log$ likelihoods between these models is distributed 
asymptotically as a $1 / 2: 1 / 2$ mixture of $\chi_{1}^{2}$ and a point mass at zero and is consequently designated $\chi_{0.1}^{2}$ (Self \& Liang, 1987). The difference between the two $\log _{10}$ likelihoods (i.e., dividing the likelihood ratio Chi square by $2 \ln 10=4.6)$, produces a lod score equivalent to the classical lod score of parametric linkage analysis (Williams \& Blangero, 1999).

Families of DZ twin pairs plus any available sibs form the main part of the data (419 families, 1,025 twins/sibs). Families of $\mathrm{MZ}$ twins were also included in the linkage analysis if they had at least one sib also assessed for eye color (83 families, 180 individuals, omitting one twin from each pair). Note that although parents were not phenotyped, their genotypes (where available) still contributed to IBD estimation for siblings.

\section{$\overline{\text { Results }}$}

The fit of the bivariate threshold model (assessed in PRELIS) to the eye color data measured on our 3 -point rating scale is good; for $438 \mathrm{DZ}$ pairs the RMSEA is $.066, P=.21$. Polychoric correlations (estimated in $\mathrm{Mx}$ ) for all 920 adolescent twin pairs who have taken part in our study so far (to October 2003) are shown for each twin group in Table $1 \mathrm{a}$ and $1 \mathrm{~b} . \mathrm{MZ}$ correlations were very close to 1 , and while there was some suggestion of shared environmental influence $(C)$ in females $\left(r_{\mathrm{dzf}}=.66\right)$, in fact the three DZ correlations do not differ significantly $\left(\chi_{2}^{2}=3.00, p=.22\right)$ and the pooled estimate of the DZ correlation is .54. The MZ and DZ correlations are in agreement with a purely additive genetic model for familial aggregation; dropping $\mathrm{C}$ from an ACE model produces a deterioration in fit of only $\chi^{2}{ }_{1}=$ $1.05(p=.31)$. Consequently, our initial model for QTL linkage analysis specified an additive QTL for which pairs that were $\mathrm{IBD}=2,1$, and 0 at a given marker locus were correlated $1,1 / 2$, and 0 respectively. However, we realise that an additive polygenic variance component may easily include individual QTL effects which behave non-additively.

The results of the linkage scan for QTLs influencing eye color are displayed in Figure 4. On the y-axis of each chromosome panel is the lod score, calculated as $\Delta \chi_{0.1}^{2} /(2 \ln 10)$, where $\Delta \chi_{0.1}^{2}$ is twice the difference in $\log$ likelihood between a model that includes the linked QTL component Q, and one which does not.

\section{Table 1a}

Polychoric Correlations and 95\% Confidence Interval for Adolescent Twins Assessed on 3-point Rating Scale for Eye Color

\begin{tabular}{lcccc}
\hline & & & \multicolumn{2}{c}{$95 \% \mathrm{Cl}$} \\
Zygosity & $N$ pairs & $r$ & Lower & Upper \\
\hline MZF & 203 & 0.988 & 0.980 & 0.993 \\
MZM & 186 & 0.999 & 0.995 & 1.000 \\
DZF & 139 & 0.655 & 0.478 & 0.778 \\
DZM & 138 & 0.451 & 0.261 & 0.607 \\
DZFM & 254 & 0.543 & 0.409 & 0.653 \\
all MZ & 389 & 0.992 & 0.987 & 0.995 \\
all DZ & 531 & 0.543 & 0.450 & 0.625 \\
Total & 920 & & & \\
\hline
\end{tabular}

\section{Table 1b}

Heterogeneity of Three DZ Correlations: $\chi_{2}^{2}=3.00, p=.22$ ACE Model Fit

\begin{tabular}{lcccccc}
\hline Model & $-2 \mathrm{LL}$ & $\Delta \chi^{2},{ }_{1}$ & $p$ & $\mathrm{~A} \%$ & $\mathrm{C} \%$ & $\mathrm{E} \%$ \\
\hline ACE & 3082.97 & & & 89.8 & 9.4 & 0.8 \\
AE & 3084.02 & 1.05 & .31 & 99.2 & & 0.8 \\
\hline
\end{tabular}

Since both MZ and DZ twin sibships are included in the dataset, we fitted an AEQ model at each marker, and compared it with an AE model, which of course needs to be fitted only once. Little difference was seen between linkage results for the two scoring systems used in the case of inconsistent ratings, so the treatment using only the nurse rating from the first visit is reported. The linkage results from this one-locus model are shown as red lines in Figure 4.

The outstanding feature of the linkage scan is the prominent peak on chromosome $15 \mathrm{q}$. Further details of the fit of models at the peak marker D15S1002, which we note is less than $1 \mathrm{cM}$ telomeric of OCA2, are shown in Table 2. The change of fit in dropping an additive QTL (Q) from an FEQ model at this marker position is $\Delta \chi_{1}^{2}=88.4$, equivalent to a lod of 19.2 . This QTL is estimated to account for $74 \%$ of total variance in eye color liability, with $18 \%$ due to familial (polygenic) background and $8 \%$ due to unique environment (of which most is probably measurement error). Since brown eye color is dominant over blue using the usual penetrance parameterization (although closer to additive under the multifactorial threshold

\section{Table 2}

Results of Fitting Additive (Q) and Dominant (D) OTLs Linked to D15S1002. Variance Components (95\% Confidence Interval) Due to Background Familial Correlation (F) and Unique Environment (E) are Also Shown as Percentages

\begin{tabular}{|c|c|c|c|c|c|c|c|c|c|c|c|c|c|}
\hline \multicolumn{2}{|c|}{ Model\# } & \multirow{2}{*}{$\frac{-2 \mathrm{LL}}{2393.54}$} & \multirow{2}{*}{$\frac{d f}{1258}$} & \multirow{2}{*}{$\frac{\text { versus \# }}{3}$} & \multirow{2}{*}{$\begin{array}{l}\Delta \chi^{2}{ }_{1} \\
15.88\end{array}$} & \multicolumn{2}{|r|}{$\mathrm{F}$} & \multicolumn{2}{|r|}{$E$} & \multicolumn{2}{|r|}{0} & \multicolumn{2}{|r|}{ D } \\
\hline FEOD & 1 & & & & & 18.2 & $(3.5-33.8)$ & 7.9 & $(4.3-13.3)$ & 73.9 & $(41.4-89.6)$ & 0.0 & $(0-21.7)$ \\
\hline FEO & 2 & 2393.54 & 1259 & 1 & 0.00 & 18.2 & $(3.5-31.9)$ & 7.9 & $(4.3-13.3)$ & 73.9 & $(58.8-89.6)$ & & \\
\hline FED & 3 & 2409.42 & 1259 & 4 & 72.54 & 43.4 & $(33.5-52.5)$ & 7.5 & $(4.1-12.9)$ & & & 49.1 & $(38.9-59.8)$ \\
\hline FE & 4 & 2481.96 & 1260 & 2 & 88.42 & 55.8 & $(48.1-62.8)$ & 44.2 & $(37.2-51.9)$ & & & & \\
\hline
\end{tabular}



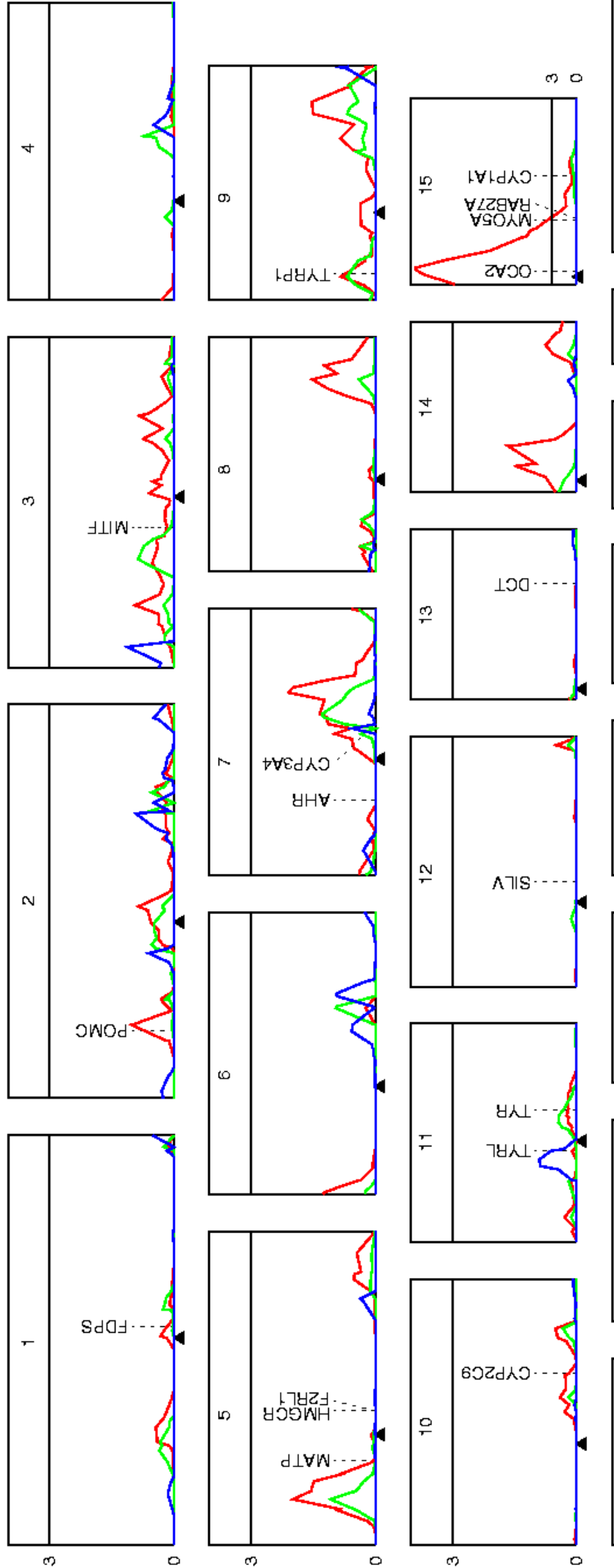
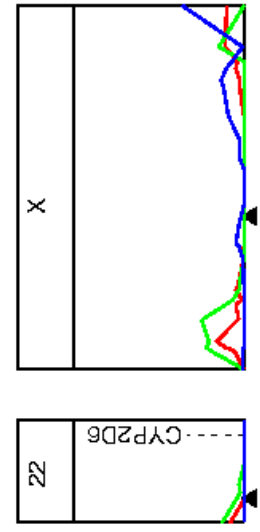

ㅊ.
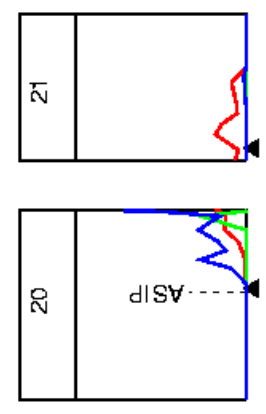

$\sigma$
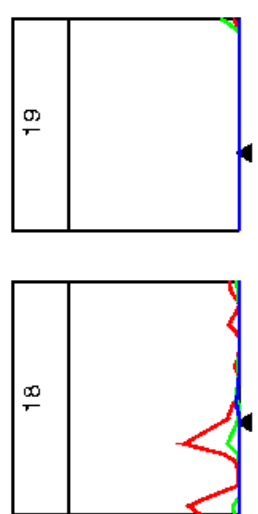

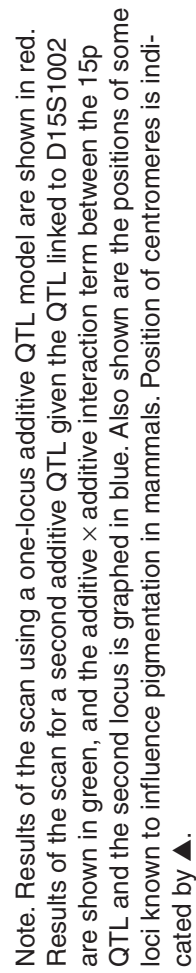

Figure 4

Results of genome-wide linkage scan for eye color measured on a 3-point scale, using variance components analysis for a multifactorial threshold model implemented in $\mathrm{Mx}$. 
model; Duffy et al., 2004), we also fitted a dominant QTL effect, D, linked to the peak marker D15S1002, by specifying loadings of 1,0 , and 0 on pairs IBD $=2$, 1 , and 0 respectively. This is in addition to the additive QTL effect Q. Dropping Q from the full model (FEQD) resulted in $\Delta \chi^{2}{ }_{1}=15.9$, whereas dropping $\mathrm{D}$ produced no change. Further, dropping $\mathrm{D}$ from the FED model resulted in $\Delta \chi^{2}{ }_{1}=72.5$. From this we conclude that, while the additive QTL model gives the better fit and results in a larger lod score, most of the linkage information is indeed coming from the IBD = 2 pairs, consistent with dominant inheritance. Our inability to resolve $\mathrm{Q}$ and $\mathrm{D}$ is due to their unavoidable confounding in the sib statistics we have available.

Given the overwhelming contribution of the $15 \mathrm{q}$ QTL to variation in eye color, we thought we might get better resolving power for secondary QTLs, by repeating the genome scan using a two-locus model in which the $15 \mathrm{q}$ linkage is always present. Initially we allowed for only an additive QTL at $15 \mathrm{q}(\mathrm{Q})$, an additive QTL at the second marker locus (R; see green lines in Figure 4), and an additive $\times$ additive $(\mathrm{Q} \times \mathrm{R})$ epistatic interaction (see blue lines in Figure 4); the coefficient for the interaction term is the product of the corresponding $\hat{\pi}_{i j}$ values at the two loci; all parameters were estimated free in each run. The most interesting feature of the results of the two-locus model is the modification of evidence for linkage from the one-locus model (see red lines in Figure 4). In most cases the evidence for linkage at secondary locations is reduced in the two-locus model, notably on $14 \mathrm{q}, 8 \mathrm{q}, 5 \mathrm{p}, 17 \mathrm{p}, 7 \mathrm{q}$ and $2 \mathrm{p}$, the latter being directly over the pro-opiomelanocortin gene (POMC). In only a couple of locations is it slightly increased (Xp, 6q). In some cases the peak location seems to shift, or be refined $(7 q, 3 p)$. However, the main impression from the two-locus analysis is that, after the $15 \mathrm{q}$ linkage was allowed for, there was very little linkage evidence for secondary QTLs affecting eye color. This was true a fortiori for additive $\times$ additive epistasis between the $15 \mathrm{q}$ QTL and a secondary locus. There was a hint of interaction at 3pter, 20qter, on $6 \mathrm{p}$ and, most interestingly, on $11 \mathrm{p}$ close to the TYRL locus. But none of these approached significance, which is not surprising given that we would expect very low power to detect such components due to confounding (Mather, 1974).

Consequently, when we fit the full two-locus model (Mather, 1974; Tiwari \& Elston, 1997), allowing for both additive and dominant effects at both the $15 \mathrm{q}$ locus and secondary locus, plus all possible pairwise interactions between them (eight parameters in all plus $\mathrm{F}$ and $\mathrm{E}$ ), it was hardly surprising that we saw virtually no change from the $\mathrm{Q}, \mathrm{R}, \mathrm{Q} \times \mathrm{R}$ model, and therefore we did not report these results. The full two-locus model is likely to be much more informative when the causal SNPs have been found and the parameters can be fitted as fixed effects, rather than random effects as here, which necessarily are detected with low power.

Also shown in Figure 4 are the locations of some genes known to be involved in mammalian pigmentation (Sturm et al., 2001), and therefore potential candidates as eye color QTLs. The peak on $15 \mathrm{q}$ lies directly over OCA2 which has already been implicated in eye color (Rebbeck et al., 2002). The large shoulder on $15 \mathrm{q}$ peak suggests that other genes influencing eye color may lie telomeric to OCA2 and we note the location of MYO5A and RAB27A in this region.

\section{Discussion}

Treating eye color as an ordinal variable with three categories, and estimating polychoric correlations under the assumption of normally distributed liability, the twin correlations we observe are compatible with a trait measured with negligible error, and almost perfect genetic additivity. The slight suggestion we saw of shared environmental effects in females is possibly due to rater bias in giving twin sisters the same rating, or possibly to assortative mating for eye color, which would be expected to increase additive genetic variance between both $\mathrm{MZ}$ and $\mathrm{DZ}$ pairs to imitate shared environmental effects. In either case, the effect was not significant in our data and might well be stochastic. It is also possible that true evidence of shared effects due to rater bias or assortative mating was reduced by confounding in the twin design with genetic dominance. Stochastic error is also the most likely explanation for the improbably high oppositesex DZ correlation for iris color in recently reported Swedish data (Larsson et al., 2003) which distorts the total DZ correlation in that sample to suggest shared environmental influence, although there is not a hint of it in the (admittedly scanty) same-sex data.

Consequently, and despite classical evidence that brown eyes are (mostly) dominant over blue, we began our linkage analysis by fitting a linked QTL inherited in an additive manner (see red lines in Figure 4). For the linkage peak on $15 \mathrm{q}$, the largest lod score (19.2) was obtained with an additive QTL model. But a lod nearly as large (15.8) was obtained with a dominant QTL model which used only approximately $25 \%$ of the sample of sib pairs who are IBD2 and discarded approximately $50 \%$ who were IBD1. Not surprisingly, given the low resolving power for nonadditive genetic effects using variance components analysis (Martin et al., 1977), the evidence for dominance versus additivity at this QTL was inconclusive, and other, more powerful methods such as segregation analysis need to be used; this will be the subject of future reports. Since the QTL at $15 \mathrm{q}$ explains $74 \%$ of the variation and since there probably is dominance at that locus, one would have expected to find a corresponding pattern of $\mathrm{MZ}$ and DZ correlations suggesting dominance. This was not the case, but Lynch and Walsh (1998) point out that despite the existence of strong dominance effects or 
epistatic interactions between the effects of genes, most of these effects are associated statistically with additive effects. Thus, the relative magnitudes of variance components (or the pattern of $\mathrm{MZ}$ and DZ twin correlations) provide little insight into the actual physiological mode of gene action (p. 87 and p. 92).

Given the overwhelming contribution of the $15 \mathrm{q}$ QTL to variation in eye color, we thought we might get better resolving power for secondary QTLs by repeating the genome scan using a two-locus model in which the $15 \mathrm{q}$ linkage was always present. In fact, this seemed to reduce the evidence for linkage at nearly all the secondary locations seen in the one-locus model. This surprised us at first, but on reflection it is possible that both false positives and genuine second loci depend upon the same coincidence of phenotype and IBD similarity as is responsible for the major QTL. Thus, eradication of a minor peak in the two-locus scan (notably, chromosome 14) does not necessarily mean that it was a false positive.

In the two-locus model, we also fitted an additive $x$ additive interaction term, but our genome scan shows no striking evidence of epistasis. Nor did this change when we allowed for more elaborate epistatic interactions by fitting the full two-locus model with additive and dominance QTL effects at both the $15 \mathrm{q}$ and secondary loci, and all four possible interaction terms. However, we recognise that the power to detect these as variance components is very weak, and await the opportunity to fit these terms as fixed effects when modifier loci of OCA2 are identified.

The study of coat color in the mouse and comparative genomic analysis with other mammals, including humans, have provided enormous insight into the genetic basis of pigmentation. More than 127 loci influencing mouse pigmentation have been described and 59 of these genes have been cloned and sequenced (Bennett \& Lamoreux, 2003). In each case a human ortholog (Jackson, 1997) has been identified and the majority of these genes have been found to be loci not only for pigmentation but also for disorders effecting other organ systems. While species comparisons have helped to identify many human pigmentation genes, it is expected that a significant number still remain to be identified and that some of these will be associated with rare genetic diseases (Spritz et al., 2003). Nevertheless, only a relatively small subset of these candidate pigmentation genes will contribute to the normal color variation within diverse human populations (Sturm et al., 1998; Sturm et al., 2001).

Pigmentation genes characterized to date fall into a limited number of functional groups effecting cellular processes such as melanocyte formation, development and differentiation, melanosomal components involved in enzymatic catalysis, structure or substrate transport, organelle biogenesis, cellular trafficking, and the regulatory pathway of pigment-type switching (Kushimoto et al., 2003; Marks \& Seabra, 2001; Swank et al., 2000). Although the power of mouse genetics is evident in identifying genes involved in coat color, less is known about the genetics of human skin and eye color. It must be noted that there are physiological differences in the cutaneous melanocyte environment between humans and mice. Murine melanocytes are located in the dermal follicles whereas human melanocytes are found in the epidermis and uveal tract. Thus the genetic basis for human skin and eye color may not be fully explicable in terms of mouse coat pigmentation genes and some of the relevant processes may only be amenable to genetic study in humans. Furthermore, with a large number of genes influencing pigmentation, some will lie under linkage peaks by chance and be irrelevant to eye color, while others with effects on eye color may lie in regions with negligible or even no linkage signal because of lack of power. In future papers we shall address linkage to skin and hair color and pleiotropic effects of loci on these three aspects of pigmentation.

Ours is the first study to use a complete genome scan in an attempt to map genes responsible for eye color. The peak on $15 \mathrm{q}$ lies directly over OCA2, which has already been implicated in eye color (Rebbeck et al., 2002). This peak has a long tail toward the telomere, suggesting that other eye color QTLs may lie there, and it is interesting that both the MYO5A and RAB27A proteins involved in melanosome trafficking (Marks \& Seabra, 2001) are located in this region.

OCA4 locus encodes a potential membrane-associated transport protein (MATP; Newton et al., 2001). The peak lod of 2.02 on $5 \mathrm{p}$ is further from OCA4 than one might expect if this were the locus responsible, although it is known that the location of peaks is subject to considerable error (Attwood \& HeardCosta, 2003; Roberts et al., 1999). The small peak $(\operatorname{lod}=1.19)$ on $2 \mathrm{p}$ lies directly over POMC, the gene encoding the precursor to the MSH ligand that binds the MC1R receptor regulating pigment type switching (Thody \& Graham, 1998), and is worthy of further investigation, as is the peak of 1.86 on $7 \mathrm{q}$, under which lies CYP3A4. The linkage peak over the known pigmentation gene TYRP1, responsible for the OCA3 albinism (Boissy \& Nordlund, 1997), on 9p is much smaller than that toward the q terminus of 9 , where there are no obvious pigmentation candidate genes. Most intriguing is the similar lack of candidates under the peak of 2.21 on $14 \mathrm{q}$, although we note that the gunmetal coat color gene RABGGTA is at $14 \mathrm{q} 11.2$ (Bennett \& Lamoreux, 2003). Smaller peaks on chromosomes 17 and 18 are in similarly candidate-free zones. It is worth noting, however, that since any other locus can explain no more than $18 \%$ of phenotypic variance, there is probably not enough power to detect the effects of other loci at conventional significance levels.

Human eye color exhibits a transitional range from light (blues) to medium (green/hazels) to dark (browns) and was demonstrated at the turn of the century to be an inherited trait (Davenport \& 
Davenport, 1907). The density and distribution of melanin granules within the stroma of the iris is responsible for eye color, with blue eyes resulting from the dilution or aberrant dispersion of the larger and more numerous melanosomes per cytoplasmic area apparent in brown eyes, and resulting in this physical difference in appearance (Eagle, 1988; Imesch et al., 1996; Wilkerson et al., 1996). It is the function of melanocytes once established in the iridial stroma, and not the embryonic development of the melanocytes migrating through the stroma of the eye, which determines iris color. Thus it is expected that genes involved in the maturation or movement of the melanosome will be major genes associated with blue eye color.

The P-protein is encoded by the mouse pink-eyed (p) dilute gene and mutations in the orthologous human OCA2 gene result in type II albinism. Other polymorphisms at this locus are associated with blue (this study) and brown eye color (Rebbeck et al., 2002). Studies in mouse melanocytes have shown that antibodies directed against the P-protein bind to the melanosomal membrane and this intracellular location suggests that it might transport a critical substance between the cytoplasm and the lumen of melanosomes (Brilliant, 2001). In situ tyrosinase activity requires an appropriate ionic environment, and it has been suggested that the P-protein may maintain an optimal melanosomal $\mathrm{pH}$ to regulate pigment formation (Ancans et al., 2003). Melanosomes from $p$-null mice have also been noted to exhibit an abnormal melanosomal ultrastructure, in addition to deficient TYR activity. In studies designed to examine the distribution of the melanosomal proteins in these cells, misrouting of the TYR protein was found (Manga et al., 2001), with accumulation of TYR in premelanosome vesicles suggesting a role for the P-protein in the processing and trafficking of melanosomal proteins (Chen et al., 2002; Toyofuku et al., 2002). Heterologous expression of recombinant P-protein in yeast has revealed a novel activity associated with the OCA2 gene product in that it can modulate arsenic sensitivity of cells, with wildtype melanocytes more sensitive to these toxic compounds than $p$-null melanocytes (Staleva et al., 2002). By using a glutathione-conjugating dye it was found that the P-protein can facilitate the vacuolar accumulation of glutathione, which could be an important regulatory event for TYR activity by imposing redox inhibition of the enzyme.

The human P-gene transcript encoded by OCA2 is divided into 24 exons covering over $267 \mathrm{~kb}$. The gene encodes an 838 amino acid open reading frame producing a $110 \mathrm{kD}$ protein that contains 12 transmembrane spanning regions; it has been located as an integral melanosomal membrane protein. At least 35 apparently non-pathogenic variant alleles of the OCA2 gene have been identified, 24 of which are exonic and of these 6 result in amino acid changes (see Albinism database www.cbc.umn.edu/tad/). Some of these polymorphisms have markedly different frequencies in different populations (Kerr et al., 2000; Lee et al., 1995). With two OCA2 coding region variants, R305W and R419Q, shown to be associated with non-blue eye colors (Rebbeck et al., 2002), the specific polymorphisms of the OCA2 gene that are associated with blue eye color remain to be identified, and that is the next stage of our research.

\section{$\overline{\text { Acknowledgments }}$}

Collection of phenotypes and DNA samples was supported by grants from the Queensland Cancer Fund (NGM, NKH), the Australian National Health and Medical Research Council (950998, 981339 and 241944; NGM), and the US National Cancer Institute (CA88363; NKH, NGM, DLD, RAS, GWM). The genome scans were supported by the Australian NHMRC's Program in Medical Genomics (NHMRC219178; NGM, GWM, DLD) and the Center for Inherited Disease Research (CIDR; Director, Dr Jerry Roberts) at The Johns Hopkins University (JMT, NGM). CIDR is fully funded through a federal contract from the National Institutes of Health to The Johns Hopkins University (Contract Number N01HG-65403). We thank Dr Cathy Hyland of the Australian Red Cross Blood Service, Brisbane, for initiating blood grouping; the twins and the staff in the Red Cell Reference Laboratory; Ann Eldridge, Marlene Grace and Anjali Henders for assistance; and the twins, their siblings, and their parents for their cooperation. We thank Dorret Boomsma and Danielle Posthuma for incisive comments on the manuscript.

\section{References}

Abecasis, G. R., Cherny, S. S., Cookson, W. O., \& Cardon, L. R. (2001a). GRR: Graphical representation of relationship errors. Bioinformatics, 17 , 742-743.

Abecasis, G. R., Cherny, S. S., \& Cardon, L. R. (2001b). The impact of genotyping error on family-based analysis of quantitative traits. European Journal of Human Genetics, 9, 130-134.

Abecasis, G. R., Cherny, S. S., Cookson, W. O., \& Cardon, L. R. (2002). Merlin - Rapid analysis of dense genetic maps using sparse gene flow trees. Nature Genetics, 30, 97-101.

Aitken, J. F., Pfitzner, J., Battistutta, D., O’Rourke, P. K., Green, A. C., \& Martin, N. G. (1996). Reliability of computer image analysis of pigmented skin lesions of Australian adolescents. Cancer, 78, 252-257.

Akey, J. M., Wang, H., Xiong, M., Wu, H., Liu., W., Shriver, M. D., et al. (2001). Interaction between the melanocortin-1 receptor and $\mathrm{P}$ genes contributes to inter-individual variation in skin pigmentation phenotypes in a Tibetan population. Human Genetics, 108, 516-520. 
Almasy, L., \& Blangero, J. (1998). Multipoint quantitativetrait linkage analysis in general pedigrees. American Journal of Human Genetics, 62, 1198-1211.

Amos, C.I. (1994). Robust variance-components approach for assessing genetic linkage in pedigrees. American Journal of Human Genetics, 54, 535-543.

Ancans, J., Flanagan, N., Hoogduijn, M. J., \& Thody, A. J. (2003). P-locus is a target for the melanogenic effects of MC-1R signaling: A possible control point for facultative pigmentation. Annals of the New York Academy of Sciences, 994, 373-377.

Attwood, L. D., \& Heard-Costa, N. L. (2003). Limits of fine mapping a quantitative trait. Genetic Epidemiology, 24, 99-106.

Bennett, D. C., \& Lamoreux, M. L. (2003). The color loci of mice - A genetic century. Pigment Cell Research, $16,333-344$.

Boehnke, M., \& Cox, N. J. (1997). Accurate inference of relationships in sib-pair linkage studies. American Journal of Human Genetics, 61, 423-429

Boissy, R. E. (1998). Extracutaneous melanocytes. In J. J. Nordlund et al. (Ed.), The pigmentary system; Physiology and pathophysiology. New York: Oxford University Press.

Boissy, R. E., \& Nordlund, J. J. (1997). Molecular basis of congenital hypopigmentary disorders in humans: a review. Pigment Cell Research, 10, 12-24.

Brilliant, M. H. (2001). The mouse p (pink-eyed dilution) and human $\mathrm{P}$ genes, oculocutaneous albinism type 2 (OCA2), and melanosomal pH. Pigment Cell Research, 14, 86-93.

Chen, K., Manga, P., \& Orlow, S. J. (2002). Pink-eyed dilution protein controls the processing of tyrosinase. Molecular Biology of the Cell, 13, 1953-1964.

Davenport, G. C., \& Davenport, C. B. (1907). Heredity of eye-color in man. Science, 26, 590-592.

Davis, S., \& Weeks, D. E. (1997). Comparison of nonparametric statistics for detection of linkage in nuclear families: Single-marker evaluation. American Journal of Human Genetics, 61, 1431-1444.

Douglas, J. A., Boehnke, M., \& Lange, K. (2000). A multipoint method for detecting genotyping errors and mutations in sibling-pair linkage data. American Journal of Human Genetics, 66, 1287-1297.

Duffy, D. L. (2002). Sib-pair version 0.99.9 [Computer program]. Brisbane, Australia: Queensland Institute of Medical Research.

Duffy, D. L., Box, N. F., Chen, W., Palmer, J. S., Montgomery, G. W., James, M. R., et al. (in press). Interactive effects of MC1R and OCA2 on melanoma risk phenotypes. Human Molecular Genetics.

Duggirala, R., Williams, J. T., Williams-Blangero, S., \& Blangero J. (1997). A variance component approach to dichotomous trait linkage analysis using a threshold model. Genetic Epidemiology, 14, 987-992.
Eagle, R. C., Jr. (1988). Iris pigmentation and pigmented lesions: An ultrastructural study. Transactions of the American Ophthalmological Society, 86, 581-687.

Eaves, L. J., Neale, M. C., \& Maes, H. (1996). Multivariate multipoint linkage analysis of quantitative trait loci. Behavior Genetics, 26, 519-525.

Eiberg, H., \& Mohr, J. (1987). Major genes of eye colour and hair colour linked to LU and SE. Clinical Genetics, 31, 186-191.

Eiberg, H., \& Mohr, J. (1996). Assignment of genes coding for brown eye colour (BEY2) and brown hair colour (HCL3) on chromosome 15q. European Journal of Human Genetics, 4, 237-241.

Epstein, M. P., Duren, W. L., \& Boehnke, M. (2000). Improved inference of relationship for pairs of individuals. American Journal of Human Genetics, 67, 1219-1231.

Ewen, K. R., Bahlo, M., Treloar, S. A., Levinson, D. F, Mowry, B., Barlow, J. W., et al. (2000). Identification and analysis of error types in high-throughput genotyping. American Journal of Human Genetics, 67, 727-736.

Fulker, D. W., \& Cherny, S. S. (1996). An improved multipoint sib-pair analysis of quantitative traits. Behavior Genetics, 26, 527-532.

Gudbjartsson, D. F., Jonasson, K., Frigge, M. L., \& Kong, A. (2000). Allegro, a new computer program for multipoint linkage analysis. Nature Genetics, 25, 12-13.

Hasstedt, S. J. (1995). Phenotypic assortative mating in segregation analysis. Genetic Epidemiology, 12, 109-127.

Imesch, P. D., Bindley, C. D., Khademian, Z., Ladd, B., Gangnon, R., Albert, D. M., et al. (1996). Melanocytes and iris color. Electron microscopic findings. Archives of Ophthalmology, 114, 443-447.

Jackson, I. J. (1997). Homologous pigmentation mutations in human, mouse and other model organisms. Human Molecular Genetics, 6, 1613-1624.

Kerr, R., Stevens, G., Manga, P., Salm, S., John, P., Haw, T., et al. (2000). Identification of $P$ gene mutations in individuals with oculocutaneous albinism in subSaharan Africa. Human Mutation, 15, 166-172.

Kong, A., Gudbjartsson, D. F., Sainz, J., Jonsdottir, G. M., Gudjonsson, S. A., Richardsson, B., et al. (2002). A high-resolution recombination map of the human genome. Nature Genetics, 31, 241-247.

Kushimoto, T., Valencia, J. C., Costin, G. E., Toyofuku, K., Watabe, H., Yasumoto, K., et al. (2003). The melanosome: An ideal model to study cellular differentiation. Pigment Cell Research, 16, 237-244.

Lange, K., Westlake, J., \& Spence, M. A. (1976). Extensions to pedigree analysis. III. Variance components by the scoring method. Annals of Human Genetics, 39, 485-491.

Lange, K., Cantor, R., Horvath, S., Perola, M., Sabatti, C., Sinsheimer, J., et al. (2001). Mendel version 4.0: A complete package for the exact genetic analysis of 
discrete traits in pedigree and population data sets. American Journal of Human Genetics, 69 (Supplement), A1886.

Larsson, M., Pedersen, N. L., \& Stattin, H. (2003). Importance of genetic effects for characteristics of the human iris. Twin Research, 6, 192-200.

Lathrop, G. M., Lalouel, J. M., Julier, C., \& Ott, J. (1984). Strategies for multilocus linkage analysis in humans. Proceedings of the National Academy of Science USA, 81, 3443-3446.

Leal, S. M. (2003). Genetic maps of microsatellite and single-nucleotide polymorphism markers: Are the distances accurate? Genetic Epidemiology, 24, 243-252.

Lee, S. T., Nicholls, R. D., Jong, M. T., Fukai, K., \& Spritz, R. A. (1995). Organization and sequence of the human $\mathrm{P}$ gene and identification of a new family of transport proteins. Genomics, 26, 354-363.

Lynch, M., \& Walsh, B. (1998). Genetics and analysis of quantitative traits. Sunderland, MA: Sinauer Associates.

Manga, P., Boissy, R. E., Pifko-Hirst, S., Zhou, B. K., \& Orlow, S. J. (2001). Mislocalization of melanosomal proteins in melanocytes from mice with oculocutaneous albinism type 2. Experimental Eye Research, 72, 695-710.

Marks, M. S., \& Seabra, M. C. (2001). The melanosome: Membrane dynamics in black and white. Nature Reviews: Molecular Cell Biology, 2, 738-748.

Martin, N. G., Eaves, L. J., Kearsey, M. J., \& Davies P. (1977). The power of the classical twin study. Heredity, 40, 97-116.

Mather, K. (1974). Non-allelic interaction in continuous variation of randomly breeding populations. Heredity, 32, 414-419.

McGregor, B., Pfitzner, J., Zhu, G., Grace, M., Eldridge, A., Pearson, J., et al. (1999). Genetic and environmental contributions to size, color, shape, and other characteristics of melanocytic naevi in a sample of adolescent twins. Genetic Epidemiology, 16, 40-53.

Miller, S. A., Dykes, D. D., \& Polesky, H. F. (1988). A simple salting out procedure for extracting DNA from human nucleated cells. Nucleic Acids Research, 16, 1215.

Neale, M. C., Boker, S. M., Xie, G., \& Maes, H. H. (2002). Mx: Statistical Modeling (6th ed.). Richmond, VA: Department of Psychiatry.

Newton, J. M., Cohen-Barak, O., Hagiwara, N., Gardner, J. M., Davisson, M. T., King, R. A., et al. (2001). Mutations in the human orthologue of the mouse underwhite gene (uw) underlie a new form of oculocutaneous albinism, OCA4. American Journal of Human Genetics, 69, 981-988.

Rebbeck, T. R., Kanetsky, P. A., Walker, A. H., Holmes, R., Halpern, A. C., Schuchter, L. M., et al. (2002). $\mathrm{P}$ gene as an inherited biomarker of human eye color. Cancer Epidemiology and Biomarkers Prevention, 11, 782-784.
Roberts, S. B., MacLean, C. J., Neale, M. C., Eaves, L. J., \& Kendler, K. S. (1999). Replication of linkage studies of complex traits: An examination of variation in location estimates. American Journal of Human Genetics, 65, 876-884.

Robins, A. H. (1991). Biological perspectives on human pigmentation. Cambridge, England; New York: Cambridge University Press.

Self, S. G., \& Liang, K. (1987). Asymptotic properties of maximum likelihood estimators and likelihood ratio tests under nonstandard conditions. Journal of the American Statistical Association, 82, 605-610.

Sobel, E., Papp, J. C., \& Lange, K. (2002). Detection and integration of genotyping errors in statistical genetics. American Journal of Human Genetics, 70, 496-508.

Spritz, R. A., Chiang, P. W., Oiso, N., \& Alkhateeb, A. (2003). Human and mouse disorders of pigmentation. Current Opinion in Genetics and Development, 13, 284-289.

Staleva, L., Manga, P., \& Orlow, S. J. (2002). Pink-eyed dilution protein modulates arsenic sensitivity and intracellular glutathione metabolism. Molecular Biology of the Cell, 13, 4206-4220.

Sturm, R. A., Box, N. F., \& Ramsay, M. (1998). Human pigmentation genetics: The difference is only skin deep. Bioessays, 20, 712-721.

Sturm, R. A., Teasdale, R. D., \& Box, N. F. (2001). Human pigmentation genes: Identification, structure and consequences of polymorphic variation. Gene, 277, 49-62.

Sunderland, E. (1956). Hair-colour variation in the United Kingdom. Annals of Human Genetics, 20, 312-333.

Swank, R. T., Novak, E. K., McGarry, M. P., Zhang, Y., Li, W., Zhang, Q., et al. (2000). Abnormal vesicular trafficking in mouse models of Hermansky-Pudlak syndrome. Pigment Cell Research, 13(Supplement 8), 59-67.

Thody, A. J., \& Graham, A. (1998). Does alpha-MSH have a role in regulating skin pigmentation in humans? Pigment Cell Research, 11, 265-274.

Tiwari, H. K., \& Elston, R. C. (1997). Linkage of multilocus components of variance to polymorphic markers. Annals of Human Genetics, 61, 253-261.

Toyofuku, K., Valencia, J. C., Kushimoto, T., Costin, G. E., Virador, V. M., Vieira, W. D., et al. (2002). The etiology of oculocutaneous albinism (OCA) type II: The pink protein modulates the processing and transport of tyrosinase. Pigment Cell Research, 15, 217-224.

Weeks, D. E., Conley, Y. P., Ferrell, R. E., Mah, T. S., \& Gorin, M. B. (2002). A tale of two genotypes: Consistency between two high-throughput genotyping centers. Genome Research, 12, 430-435.

Wilkerson, C. L., Syed, N. A., Fisher, M. R., Robinson, N. L., Wallow, I. H., \& Albert, D. M. (1996). 
Melanocytes and iris color. Light microscopic findings. Archives of Ophthalmology, 114, 437-442.

Williams, J. T., \& Blangero, J. (1999). Comparison of variance components and sibpair-based approaches to quantitative trait linkage analysis in unselected samples. Genetic Epidemiology, 16, 113-134.

Wright, M., de Geus, E., Ando, J., Luciano, M., Posthuma, D., Ono, Y., et al. (2001). Genetics of cog- nition: Outline of a collaborative twin study. Twin Research, 4, 48-56.

Zhu, G., Duffy, D. L., Eldridge, A., Grace, M., Mayne, C., O'Gorman, L., et al. (1999). A major quantitativetrait locus for mole density is linked to the familial melanoma gene CDKN2A: A maximum-likelihood combined linkage and association analysis in twins and their sibs. American Journal of Human Genetics, 65, 483-492. 